\title{
Measuring Feature Diversity in Native Language Identification
}

\author{
Shervin Malmasi \\ Centre for Language Technology \\ Macquarie University \\ Sydney, NSW, Australia \\ shervin.malmasi@mq.edu.au
}

\author{
Aoife Cahill \\ Educational Testing Service \\ 660 Rosedale $\mathrm{Rd}$ \\ Princeton, NJ 08541, USA \\ acahillets.org
}

\begin{abstract}
The task of Native Language Identification (NLI) is typically solved with machine learning methods, and systems make use of a wide variety of features. Some preliminary studies have been conducted to examine the effectiveness of individual features, however, no systematic study of feature interaction has been carried out. We propose a function to measure feature independence and analyze its effectiveness on a standard NLI corpus.
\end{abstract}

\section{Introduction}

Researchers in Second Language Acquisition (SLA) investigate the multiplex of factors that influence our ability to acquire new languages and chief among these is the role of the learner's mother tongue. This core factor has recently been studied in the task of Native Language Identification (NLI), which aims to infer the native language (L1) of an author based on texts written in a second language (L2). Machine Learning methods are usually used to identify language use patterns common to speakers of the same L1 (Tetreault et al., 2012). While NLI has applications in security, most research has a strong linguistic motivation relating to language teaching and learning. In this context, by identifying L1-specific language usage and error patterns, NLI can be used to better understand SLA and develop teaching methods, instructions and learner feedback that is tailored to their mother tongue (Malmasi and Dras, 2014b).

Although researchers have employed tens of feature types, no effort has been made to measure the overlap of information they capture. Results from previous studies show that while some feature types yield similar accuracies independently, combining them can improve performance (Brooke and Hirst,
2012). This indicates that the information they capture is diverse, but how diverse are they and how can we measure the level of independence between the feature types?

This is a question that has not been tackled in NLI, despite researchers having examined numerous feature types to date. We examine one approach to measuring the degree of diversity between features and perform several analyses based on the results.

\section{Data and Methodology}

We use the ToEfL11 corpus (Blanchard et al., 2013) released with the 2013 NLI shared task (Tetreault et al., 2013). It includes 12,100 learner texts from 11 L1 groups, divided into train, dev. and test sets.

We use a linear Support Vector Machine ${ }^{1}$ to perform multi-class classification in our experiments.

We experiment with a wide range of previously used syntactic and lexical features: Adaptor Grammars (AG) (Wong et al., 2012), character $n$-grams (Tsur and Rappoport, 2007), ${ }^{2}$ Function word unigrams and bigrams (Malmasi et al., 2013), Lemma and Word $n$-grams, CFG Production Rules (Wong and Dras, 2011), Penn Treebank (PTB) part-of-speech $n$-grams, RASP part-ofspeech $n$-grams (Malmasi et al., 2013), Stanford Dependencies with POS transformations (Tetreault et al., 2012), and Tree Substitution Grammar (TSG) fragments (Swanson and Charniak, 2012). The individual feature accuracies ${ }^{3}$ are shown in Figure $1 .{ }^{4}$

\footnotetext{
${ }^{1}$ We use LIBLINEAR. Additional preliminary experiments with alternative learners yielded similar results.

${ }^{2}$ We treat character $n$-grams as lexical features in this work but restrict our investigation to 1-3-grams. Recent work has also shown improvements from longer sequences (Jarvis et al., 2013; Ionescu et al., 2014).

${ }^{3}$ Obtained by training on the ToEFL11 train and development sets and evaluating on the test set.

${ }^{4}$ Listed in alphabetical order.
} 


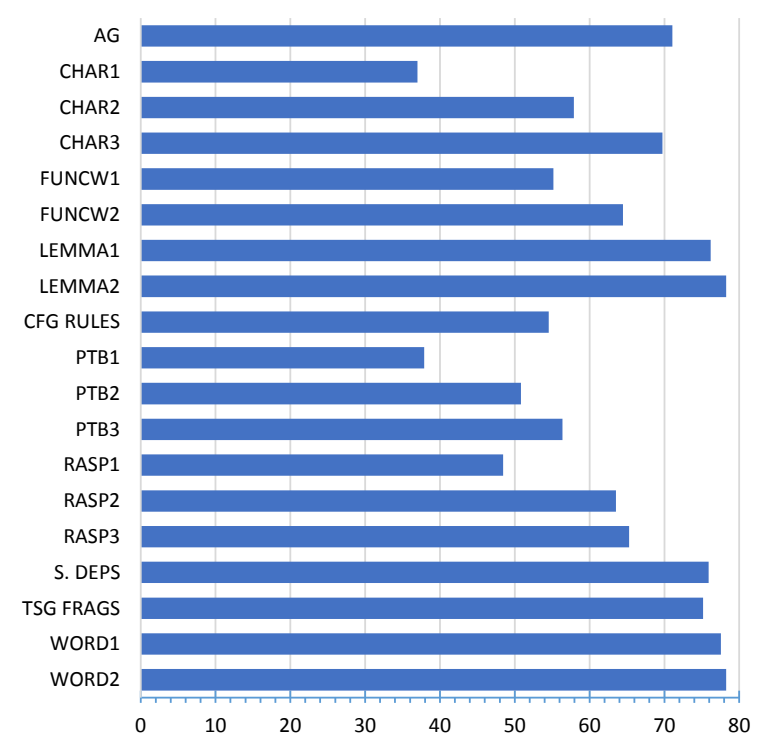

Figure 1: Individual classification accuracy for each one of our features on the TOEFL11 test set.

\section{Measuring Feature Diversity}

An ablation study is a common approach in machine learning that aims to measure the contribution of each feature in a multi-component system. This ablative analysis is usually carried out by measuring the performance of the entire system with all components (i.e. features) and then progressively removing the components one at a time to measure how the performance degrades. 5

While useful for estimating the potential contribution of a component, this type of analysis does not directly inform us about the pairwise relation between any two given components. This shortcoming has been noted by other researchers, e.g. Wellner et al. (2009, p. 122), and highlights the need to quantify the overlap between any two given components in a system. Our approach to quantifying the diversity between two feature types is based on measuring the level of agreement between the two for predicting labels on the same set of documents. Here, we aim to examine feature differences by holding the classifier parameters and data constant.

Past research suggests that Yule's Q-coefficient statistic (Yule, 1912) is a useful measure of pairwise dependence between two classifiers (Kuncheva et al., 2003). This notion of dependence relates to complementarity and orthogonality, and is an important factor in combining classifiers (Lam, 2000).

Yule's Q statistic is a correlation coefficient for binary measurements and can be applied to classi-

\footnotetext{
${ }^{5}$ Other variations exist, e.g. compare Richardson et al. (2006) and Wellner et al. (2009)
}

fier outputs for each data point where the output values represent correct (1) or incorrect (0) predictions made by that learner. Each classifier $C_{i}$ produces a result vector $y_{i}=\left[y_{i, 1}, \ldots, y_{i, N}\right]$ for a set of $N$ documents where $y_{i, j}=1$ if $C_{i}$ correctly classifies the $j^{\text {th }}$ document, otherwise it is 0 . Given these output vectors from two classifiers $C_{i}$ and $C_{k}$, a $2 \times 2$ contingency table can be derived, as shown in Table 1.

$\begin{array}{lcc} & C_{k} \text { Correct } & C_{k} \text { Wrong } \\ C_{i} \text { Correct } & N^{11} & N^{10} \\ C_{i} \text { Wrong } & N^{01} & N^{00}\end{array}$

Table 1: Contingency table for two classifiers.

Here $N^{11}$ is the frequency of items that both classifiers predicted correctly, $N^{00}$ where they were both wrong, and so on. The Q-coefficient for the two classifiers can then be calculated as:

$$
Q_{i, k}=\frac{N^{11} N^{00}-N^{01} N^{10}}{N^{11} N^{00}+N^{01} N^{10}}
$$

This distribution-free association measure ${ }^{6}$ is based on taking the products of the diagonal cell frequencies and calculating the ratio of their difference and sum. Q ranges between -1 to +1 , where -1 signifies negative association, 0 indicates no association (independence) and +1 means perfect positive correlation (dependence).

Here our classifiers are always of the same type, a linear SVM, but they are trained with different features on the same data, allowing us to measure the dependence between feature types themselves.

\section{Results}

The matrix of the Q-coefficients for all features is shown graphically in Figure 2. The most discernible feature is the red cluster in the bottom left of the matrix. This region covers the correlations between syntactic and lexical features, showing that they differ the most.

Another interesting aspect is the strong correlations between the lexical features, shown by the clustering of high values in the bottom right corner. It also shows that character $n$-grams capture similar information to word unigrams and bigrams. Even character unigrams - the lowest performing lexical feature - show much stronger dependence with word unigrams than other syntactic features. Additionally, the high values in the bottom middle section

\footnotetext{
${ }^{6}$ This is equivalent to the $2 \times 2$ version of Goodman and Kruskal's gamma measure for ordinal variables.
} 


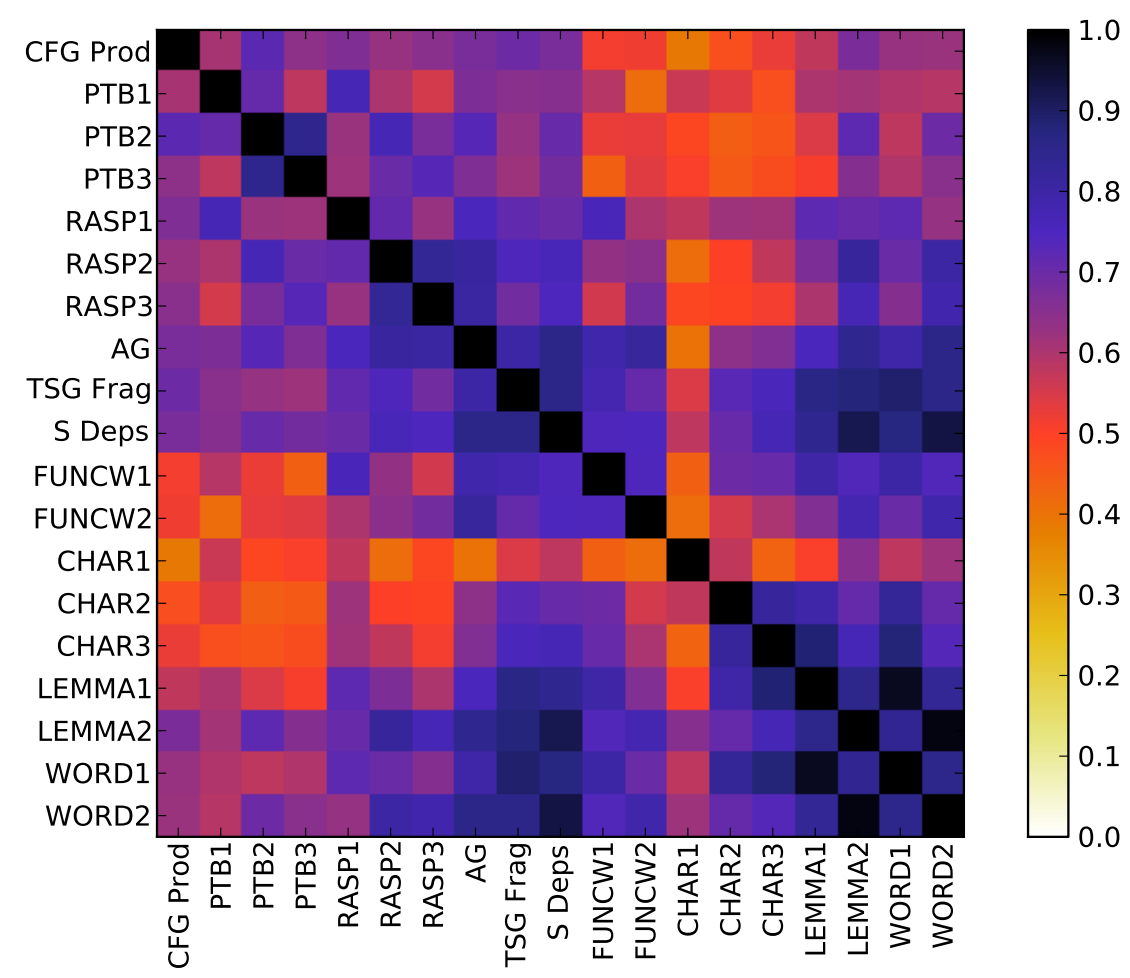

Figure 2: The Q-coefficient matrices of our feature set. The matrices are displayed as heat maps.

of the matrix show that Stanford Dependencies and TSG fragments largely capture the same information as Word and Lemma bigrams. These issues are explored further in $\S 5$.

In contrast to the lexical features, the syntactic ones show much lower inter-correlation levels, evidenced by lower values in the top left corner and absence of a visible cluster. This seems to indicate that there is greater diversity among these features.

Such analyses can help us better understand the linguistic properties of features and guide interpretation of the results. This knowledge can also be useful in creating classifier ensembles. One goal in creating such committee-based classifiers is the identification of the most diverse independent learners and this method can be applied to that end. To assess this, we also measure the accuracy for all 171 possible feature pair combinations $f_{i}$ and $f_{j}$ in our feature set. Each pair is combined in a weighted sum ensemble classifier (Malmasi et al., 2013) and run against the TOEFL11 test set. For each pair we also calculate the relative increase over only using the more accurate feature of the two $;^{7}$ this measures

\footnotetext{
${ }^{7}$ The relative increase is defined as:

Accuracy $_{f_{i}+f_{j}}-\max \left(\right.$ Accuracy $_{f_{i}}$, Accuracy $\left._{f_{j}}\right)$

An alternative metric here for this could be the "Oracle" baseline used by Malmasi et al. (2015).
}

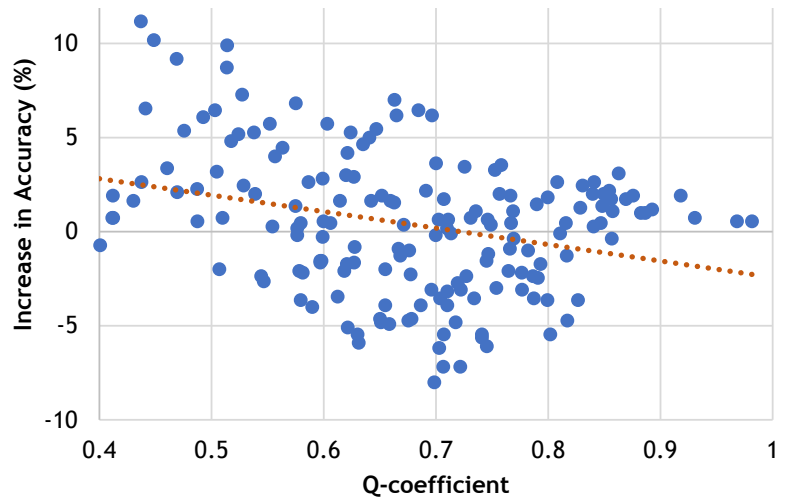

Figure 3: Scatterplot of the Q-coefficient vs relative increase in accuracy for all 171 feature pairs.

the net effect of combining the two: positive for improvements and negative for degradation.

The increase for each pair is compared against the Q-coefficient, and Pearson's correlation for the two variables shows a medium, statistically significant negative correlation $(r=-.303, p=.000)$. A scatterplot is shown in Figure 3, where we observe that almost all feature pairs with $Q<0.5$ yielded a net increase while many pairs with $Q>0.6$ resulted in performance degradation.

The measure is particularly useful when comparing features with similar individual accuracy to identify sets with the highest diversity. This is because 


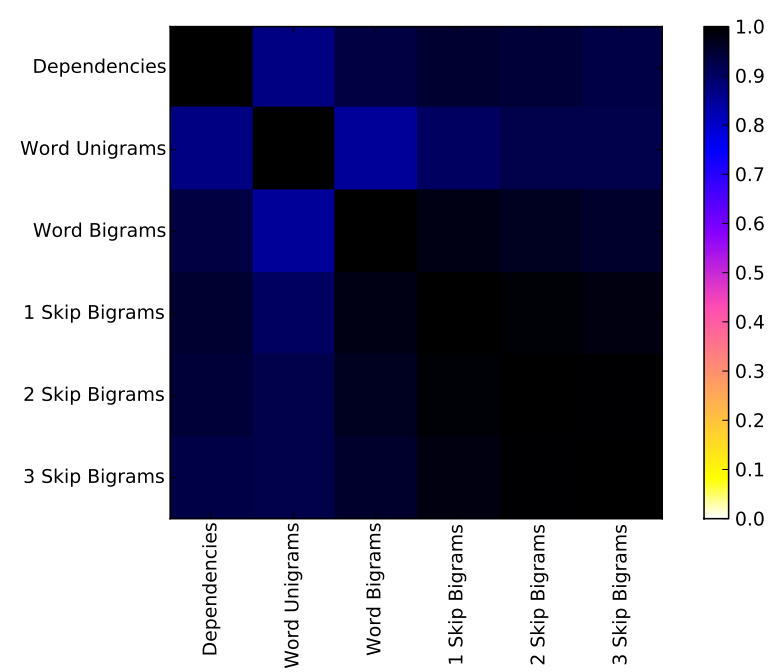

Figure 4: The Q-coefficient matrix for dependencies, word $n$-grams and skip-grams.

diversity itself cannot be the sole criterion for feature selection; a weak feature such as character unigrams will be very diverse to a strong one like POS $n$-grams but this does not ipso facto make it a good feature and we must also consider accuracy.

\section{Analyzing Words and Dependencies}

Grammatical dependencies have been found to be a very useful NLI feature and thought to capture a "more abstract representation of syntactic structures" (Tetreault et al., 2012; Bykh and Meurers, 2014). Accordingly, we were initially surprised to find the high correlation between dependencies and word bigrams $(Q=0.93)$. However, this relation may not be unexpected after all.

One source of supporting evidence comes from examining dependency distances. Using English data, ${ }^{8}$ Liu (2008) reports a Mean Dependency Distance (MDD) of 2.54 with $51 \%$ of the dependencies being adjacent and thus also captured by word bigrams. This also suggests that we can capture more of this information by considering non-adjacent tokens. We test this hypothesis by using $k$-skip word bigrams (Guthrie et al., 2006) as classification features, with $k=1-3$.

The 1-skip bigrams yield an accuracy of $79.3 \%$ on the TOEFL11 test set, higher than either word bigrams or Stanford Dependencies. The 2- and 3skip grams achieve $78.4 \%$ and $77.9 \%$. The matrix of Q-coefficients for these features is shown in Figure 4 , showing that the 1-skip word bigrams feature is the closest to the dependencies feature with a Q-

\footnotetext{
${ }^{8} 120 \mathrm{k}$ sentences averaging 21 tokens each.
}

coefficient of 0.96 . It is also the closest to standard word unigrams and bigrams with Q-coefficients of 0.91 and 0.97 , respectively.

These results suggest that skip-grams are a very useful feature for NLI. ${ }^{9}$ They could also be used as a substitute for dependencies in scenarios where running a full parser may not be feasible, e.g. realtime data processing. Moreover, with NLI being investigated with other languages (Malmasi and Dras, 2014a), this feature can be a good approximation of the dependencies feature for low-resourced languages without an accurate parser. However, results may vary by language and possibly genre (Liu, 2008). We also note that the skip-gram feature space grows prodigiously as $k$ increases.

Another related issue is whether sub-lexical character $n$-grams are independent of word features. Previously, Tsur and Rappoport (2007) hypothesized that these $n$-grams are discriminative due to writer choices "strongly influenced by the phonology of their native language". Nicolai and Kondrak (2014) also investigate the source of L1 differences in the relative frequencies of character bigrams. They propose an algorithm to identify the most discriminative words and subsequently, the bigrams corresponding to these words. They found that removing a small set of highly discriminative words greatly degrades the accuracy of a bigrambased classifier. Based on this they conclude that bigrams capture differences in word usage and lexical transfer rather than L1 phonology. Evidence from our analysis also points to a similar pattern with the predictions of character bigrams and trigrams being strongly correlated with word and lemma unigrams.

Such lexical transfer effects have been previously noted by others (Odlin, 1989). The effects are mediated not only by cognates and word form similarities, but also semantics and meanings. We also examine the link between L1 and word usage.

Using the Etymological WordNet ${ }^{10}$ database (de Melo, 2014), we extracted two lists of English words with either Old English (508 words) or Latin origins (1,310 words). These words were used as unigram features to train two classifiers. The F1-scores for classification on ToEFL11 are shown in Figure 5. The Old English words, with their West Germanic roots, yield the best results for classifying German data. Conversely, the Latinate features achieve the

\footnotetext{
${ }^{9}$ Hladka et al. (2013) and Henderson et al. (2013) previously used a skip-gram variant that did not include 0 skips as per (Guthrie et al., 2006) and did not improve accuracy.

${ }^{10} \mathrm{http}: / / \mathrm{www} 1$. icsi.berkeley.edu/\%7edemelo/etymwn/
} 


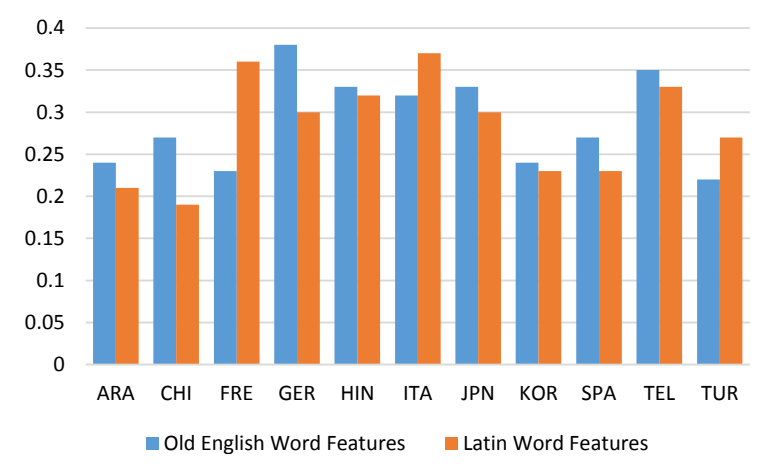

Figure 5: F1-scores for classifying L1 using English words with Old English or Latin origins.

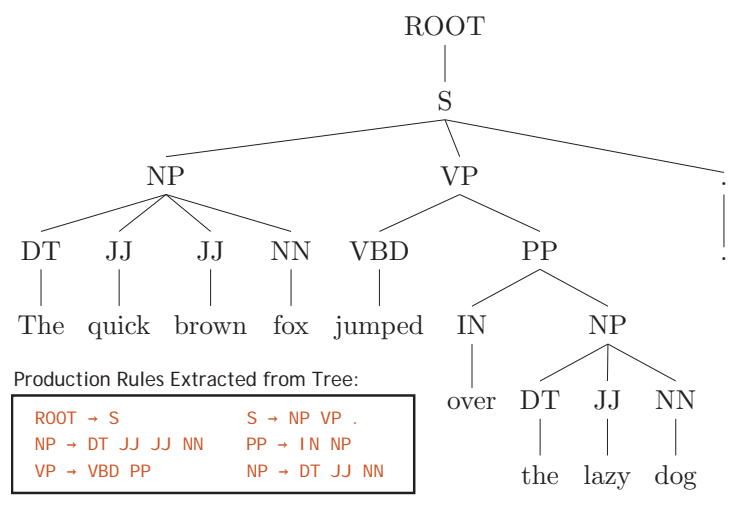

Figure 6: A constituent parse tree for an example sentence along with the context-free grammar production rules which can be extracted from it.

best results for Italian followed by French, both languages descended from Latin.

This experiment, albeit limited in scope, provides some empirical evidence suggesting that small sets of words can capture lexical transfer effects potentially mediated by L1 similarity and cognates.

\section{Parent-Annotated CFG Rules}

As demonstrated by our results, CFG production rules are a diverse syntactic feature with good accuracy. This feature type is processed by first generating constituent parses for each sentence and then extracting its production rules, ${ }^{11}$ excluding lexicalizations. Each rule is then used as a feature. Figure 6 illustrates this with an example tree and its rules. They have been successfully used in NLI (Wong and Dras, 2011) and in this section we experiment with a new extension of this feature type previously not applied to NLI.

Parent-annotated PCFG models have previously been applied in parsing and shown to yield improved

\footnotetext{
${ }^{11}$ These are the phrase structure rules used to generate constituent parts of sentences, such as noun phrases.
}
$\mathrm{ROOT} \rightarrow \mathrm{S}^{\wedge}<\mathrm{ROOT}>$
$\mathrm{VP} \wedge<\mathrm{S}>\quad \rightarrow$ VBD $\mathrm{PP}^{\wedge}<\mathrm{VP}>$
$\mathrm{S}^{\wedge}<\mathrm{ROOT}>\rightarrow \mathrm{NP} \wedge<\mathrm{S}>\mathrm{VP} \wedge<\mathrm{S}>$.
$\mathrm{PP} \wedge<\mathrm{VP}>\rightarrow$ IN $N \mathrm{P} \wedge<\mathrm{PP}>$
$\mathrm{NP} \wedge<\mathrm{S}>\rightarrow \mathrm{DT}$ JJ JJ NN
$\mathrm{NP} \wedge<\mathrm{PP}>\rightarrow$ DT JJ NN

Figure 7: Parent-annotated CFG rules from Fig. 4.

results over other models (Johnson, 1998). In this experiment we apply this feature to NLI and evaluate whether it can provide any improvement over standard production rule models.

This feature involves a modification of the linguistic tree representation, appending the category of each node's parent as additional contextual information (Johnson, 1998, p. 623). This transformation can be described as adding "pseudo contextsensitivity" (Charniak and Carroll, 1994). Figure 7 shows the parent-annotated CFG rule features extracted from the tree shown in Figure 6.

Testing this feature on the TOEFL11 test set, we achieve an accuracy of $55.6 \%, \mathrm{a}+1.3 \%$ increase over the standard CFG rules feature. Analyzing feature diversity, we observe a Q-coefficient of 0.92 between the two CFG rule based features. These results show that parent annotation leads to a sizeable increase in accuracy and also a notable change in diversity levels.

Although these initial results suggest that this is a useful feature, more testing with other data can help determine if these patterns hold across corpora (Malmasi and Dras, 2015). This additional information could also help in other tasks such as language transfer hypothesis formulation (Malmasi and Dras, 2014b) through the examination of more specific environmental contexts for features.

We leave to future work the investigation of improved ensemble classifiers that would be informed by the results of this study. The exploration of other linguistic tree representations and transformations, including Chomsky Normal Form, is another avenue for future work.

\section{Conclusion}

In this work we examined a method for measuring feature diversity in NLI and highlighted several interesting trends. We demonstrated how this analysis can be used to better understand the information captured by features and used it to examine the relationship between lexical features. We show that a variant of 1-skip bigrams can in fact be a useful feature and also proposed a new NLI feature, parentannotated CFG rules, showing how feature diversity can guide feature engineering. 


\section{Acknowledgments}

We would like to thank the anonymous reviewers for their helpful comments. We would also like to thank Keelan Evanini, Yoko Futagi and Jidong Tao for their thoughtful suggestions for improving this work.

\section{References}

Daniel Blanchard, Joel Tetreault, Derrick Higgins, Aoife Cahill, and Martin Chodorow. 2013. TOEFL11: A Corpus of Non-Native English. Technical report, Educational Testing Service.

Julian Brooke and Graeme Hirst. 2012. Robust, Lexicalized Native Language Identification. In Proceedings of COLING 2012, pages 391-408, Mumbai, India, December. The COLING 2012 Organizing Committee.

Serhiy Bykh and Detmar Meurers. 2014. Exploring Syntactic Features for Native Language Identification: A Variationist Perspective on Feature Encoding and Ensemble Optimization. In Proceedings of COLING 2014, the 25th International Conference on Computational Linguistics: Technical Papers, pages 19621973, Dublin, Ireland, August. Dublin City University and Association for Computational Linguistics.

Eugene Charniak and Glenn Carroll. 1994. Contextsensitive statistics for improved grammatical language models. In AAAI, pages 728-733.

Gerard de Melo. 2014. Etymological wordnet: Tracing the history of words. In Proceedings of the Ninth International Conference on Language Resources and Evaluation (LREC-2014). European Language Resources Association (ELRA).

David Guthrie, Ben Allison, Wei Liu, Louise Guthrie, and Yorick Wilks. 2006. A Closer Look at Skip-gram Modelling. In Proceedings of the Fifth International Conference on Language Resources and Evaluation (LREC 2006), pages 1222-1225, Genoa, Italy.

John Henderson, Guido Zarrella, Craig Pfeifer, and John D. Burger. 2013. Discriminating Non-Native English with 350 Words. In Proceedings of the Eighth Workshop on Innovative Use of NLP for Building Educational Applications, pages 101-110, Atlanta, Georgia, June. Association for Computational Linguistics.

Barbora Hladka, Martin Holub, and Vincent Kriz. 2013. Feature Engineering in the NLI Shared Task 2013: Charles University Submission Report. In Proceedings of the Eighth Workshop on Innovative Use of NLP for Building Educational Applications, pages 232241, Atlanta, Georgia, June. Association for Computational Linguistics.
Radu Tudor Ionescu, Marius Popescu, and Aoife Cahill. 2014. Can characters reveal your native language? A language-independent approach to native language identification. In Proceedings of the 2014 Conference on Empirical Methods in Natural Language Processing (EMNLP), pages 1363-1373, Doha, Qatar, October. Association for Computational Linguistics.

Scott Jarvis, Yves Bestgen, and Steve Pepper. 2013. Maximizing Classification Accuracy in Native Language Identification. In Proceedings of the Eighth Workshop on Innovative Use of NLP for Building Educational Applications, pages 111-118, Atlanta, Georgia, June. Association for Computational Linguistics.

Mark Johnson. 1998. PCFG models of linguistic tree representations. Computational Linguistics, 24(4):613-632.

Ludmila I Kuncheva, Christopher J Whitaker, Catherine A Shipp, and Robert PW Duin. 2003. Limits on the majority vote accuracy in classifier fusion. Pattern Analysis \& Applications, 6(1):22-31.

Louisa Lam. 2000. Classifier combinations: implementations and theoretical issues. In Multiple classifier systems, pages 77-86. Springer.

Haitao Liu. 2008. Dependency distance as a metric of language comprehension difficulty. Journal of Cognitive Science, 9(2):159-191.

Shervin Malmasi and Mark Dras. 2014a. Chinese Native Language Identification. Proceedings of the 14th Conference of the European Chapter of the Association for Computational Linguistics.

Shervin Malmasi and Mark Dras. 2014b. Language Transfer Hypotheses with Linear SVM Weights. In Proceedings of the 2014 Conference on Empirical Methods in Natural Language Processing (EMNLP), Doha, Qatar, October. Association for Computational Linguistics.

Shervin Malmasi and Mark Dras. 2015. Large-scale Native Language Identification with Cross-Corpus Evaluation. In Proceedings of NAACL-HLT 2015, Denver, Colorado, June. Association for Computational Linguistics.

Shervin Malmasi, Sze-Meng Jojo Wong, and Mark Dras. 2013. NLI Shared Task 2013: MQ Submission. In Proceedings of the Eighth Workshop on Innovative Use of NLP for Building Educational Applications, pages 124-133, Atlanta, Georgia, June. Association for Computational Linguistics.

Shervin Malmasi, Joel Tetreault, and Mark Dras. 2015. Oracle and Human Baselines for Native Language Identification. In Proceedings of the Tenth Workshop on Innovative Use of NLP for Building Educational Applications, Denver, Colorado, June. Association for Computational Linguistics. 
Garrett Nicolai and Grzegorz Kondrak. 2014. Does the phonology of L1 show up in L2 texts? In Proceedings of The 52nd Annual Meeting of the Association for Computational Linguistics (ACL), pages 854-859.

Terence Odlin. 1989. Language Transfer: Crosslinguistic Influence in Language Learning. Cambridge University Press, Cambridge, UK.

Matthew Richardson, Amit Prakash, and Eric Brill. 2006. Beyond PageRank: machine learning for static ranking. In Proceedings of the 15th international conference on World Wide Web, pages 707-715. ACM.

Benjamin Swanson and Eugene Charniak. 2012. Native Language Detection with Tree Substitution Grammars. In Proceedings of the 50th Annual Meeting of the Association for Computational Linguistics (Volume 2: Short Papers), pages 193-197, Jeju Island, Korea, July. Association for Computational Linguistics.

Joel Tetreault, Daniel Blanchard, Aoife Cahill, and Martin Chodorow. 2012. Native Tongues, Lost and Found: Resources and Empirical Evaluations in Native Language Identification. In Proceedings of COLING 2012, pages 2585-2602, Mumbai, India, December. The COLING 2012 Organizing Committee.

Joel Tetreault, Daniel Blanchard, and Aoife Cahill. 2013. A Report on the First Native Language Identification Shared Task. In Proceedings of the Eighth Workshop on Innovative Use of NLP for Building Educational Applications, pages 48-57, Atlanta, Georgia, June. Association for Computational Linguistics.

Oren Tsur and Ari Rappoport. 2007. Using Classifier Features for Studying the Effect of Native Language on the Choice of Written Second Language Words. In Proceedings of the Workshop on Cognitive Aspects of Computational Language Acquisition, pages 9-16, Prague, Czech Republic, June. Association for Computational Linguistics.

Ben Wellner, James Pustejovsky, Catherine Havasi, Anna Rumshisky, and Roser Sauri. 2009. Classification of discourse coherence relations: An exploratory study using multiple knowledge sources. In Proceedings of the 7th SIGdial Workshop on Discourse and Dialogue, pages 117-125. Association for Computational Linguistics.

Sze-Meng Jojo Wong and Mark Dras. 2011. Exploiting Parse Structures for Native Language Identification. In Proceedings of the 2011 Conference on Empirical Methods in Natural Language Processing, pages 1600-1610, Edinburgh, Scotland, UK., July. Association for Computational Linguistics.

Sze-Meng Jojo Wong, Mark Dras, and Mark Johnson. 2012. Exploring Adaptor Grammars for Native Language Identification. In Proc. Conf. Empirical Methods in Natural Language Processing (EMNLP), pages 699-709.
George Udny Yule. 1912. On the methods of measuring association between two attributes. Journal of the Royal Statistical Society, pages 579-652. 